BULL. AUSTRAL. MATH. SOC.

\title{
RECURRENCE RELATIONS IN A MODULAR REPRESENTATION ALGEBRA
}

\author{
J-C. RenAud
}

\begin{abstract}
In 1978 Almkvist and Fossum examined the decomposition of the exterior powers of basis modules in the modular representation algebra of a cyclic group of prime order. In particular they developed an isomorphism between these exterior powers and terms of binomial coefficient type in the algebra.

We derive several recurrence relations for these terms.
\end{abstract}

\section{Introduction}

Let $G$ be a cyclic group of order $p$ and $K$ a field of characteristic $p$. Let $v_{n}, n=1, \ldots, p$, be the standard indecomposable $(K, G)$-modules of $K$-dimension $n$.

Form the algebra $A_{p, 1}$ with basis $\left\{V_{1}, \ldots, v_{p}\right\}$ over the real field, with direct sum and tensor product operations. (For further details, see [1] and [2].)

Products in $A_{p, 1}$ can be evaluated using the formula given in Theorem 1 in [2]:

For $0 \leq r \leq s \leq p$,

$$
v_{p} X V_{s}=\sum_{i=1}^{c} V_{s-p+2 i-1}+(p-c) V_{p}
$$

Received 22 March 1982. 
where

$$
c=\left\{\begin{array}{lll}
r & \text { if } \quad r+s \leq p, \\
p-s & \text { if } \quad r+s \geq p .
\end{array}\right.
$$

In [1], Almkvist and Fossum examine the exterior powers of the $V_{n}$, and find these to be isomorphic to terms in $A_{p, 1}$ which can be expressed using the formula

$$
\Lambda^{r}\left(V_{n}\right)=\left(\begin{array}{c}
V_{n} \\
V_{r}
\end{array}\right)
$$

where the binomial expression is defined, for $1 \leq r \leq n$, by

$$
\left(\begin{array}{l}
V_{n} \\
V_{r}
\end{array}\right)=\frac{V_{n} \cdot V_{n-1} \cdots V_{n-r+1}}{V_{r} \cdot V_{p-1} \cdots V_{1}}
$$

with $\Lambda^{0}\left(V_{n}\right)=V_{1}, n \geq 1$.

They show that this quotient is in fact a term in $A_{p, I}$ whose expansion has coefficients which are partition values.

We derive several recurrence relations for the exterior powers, two of which are particularly well suited for calculating the coefficients of the expansions. The proofs are direct, using the product decomposition formula in Theorem 1 in [2]: alternate proofs via Chebyshev polynomial results are possible, but appear longer and clumsier because of difficulties with notation.

\section{Preliminary lemmas}

LEMMA 1. For $1 \leq(a, b) \leq p$, with $a+b-1 \leq p$,

$$
V_{a} X V_{b}-V_{a-1} X V_{b-1}=V_{a+b-1}
$$

Proof. From the symmetry of the formula we can assume $a \leq b$. Now by the decomposition formula,

$$
\begin{aligned}
v_{a} X V_{b}-v_{a-1} X V_{b-1} & =\sum_{i=1}^{a} v_{b-a+2 i-1}-\sum_{i=1}^{a-1} V_{b-a+2 i-1} \\
& =\dot{v}_{a+b-1} .
\end{aligned}
$$


In the following two lemmas, allow $V_{-k}=-V_{k}$.

LEMMA 2. (a) For $1 \leq(a, b)<p$, with $a+b \leq p$,

$$
\left(V_{a+1}-V_{a-1}\right) X V_{b}=V_{a+b}+V_{b-a} \text {. }
$$

(b) For $1 \leq(a, b)<p$, with $a+b \geq p$,

$$
\left(v_{a+1}-V_{a-1}\right) X V_{b}=2 V_{p}+V_{b-a}-V_{2 p-(a+b)}
$$

Proof. Direct application of the product decomposition formula in the three cases $a<b, a=b$ and $a>b$ is sufficient.

LEMMA 3. For $I \leq(a, b) \leq p$, with $a+b-1 \leq p$,

$$
V_{a} X V_{a-1}-V_{b} X V_{b-1}=V_{a+b-1} X V_{a-b} \text {. }
$$

Proof. Apply the formula to each of the three products and compare expansions.

\section{The recurrence relations}

THEOREM 1. FOr $1 \leq p<n \leq p$,

$$
\Lambda^{r}\left(V_{n}\right)=V_{r+1} \Lambda^{r}\left(V_{n-1}\right)-V_{n-r-1} \Lambda^{r-1}\left(V_{n-1}\right)
$$

Proof. Expansion of the right-hand terms, using Almkvist and Fossum's binomial result, leads to the expression

$$
\begin{aligned}
V_{r+1}\left(\begin{array}{c}
V_{n-1} \\
V_{r}
\end{array}\right)-V_{n-r-1}\left(\begin{array}{c}
V_{n-1} \\
V_{r-1}
\end{array}\right) & =\frac{V_{n-1} \ldots V_{n-r+1}}{V_{r} \cdots V_{1}}\left\{V_{r+1} V_{n-r}-V_{n-r-1} V_{r}\right\} \\
& =\frac{V_{n-1} \cdots V_{n-r+1}}{V_{r} \cdots V_{1}} \cdot V_{n} \text { on applying Lemma } 1 \\
& =\left(\begin{array}{c}
V_{n} \\
V_{r}
\end{array}\right)=\Lambda^{r}\left(V_{n}\right)
\end{aligned}
$$

as required.

Note that this is not a particularly useful form for computation of the linear expansion of the powers. However, elementary manipulation of the result is possible: clearly, $\Lambda^{r}\left(v_{n}\right)=\Lambda^{n-r}\left(v_{n}\right)$, and hence we have 
COROLLARY 1. $\Lambda^{r}\left(v_{n}\right) \cdot=V_{n-r+1} \Lambda^{r-1}\left(V_{n-1}\right)-V_{r-1} \Lambda^{r}\left(V_{n-1}\right)$

We can now combine these two results into the useful form COROLLARY 2.

$$
\Lambda^{r}\left(V_{n}\right)=\frac{3}{2}\left\{V_{r+1}-V_{p-1}\right\} \Lambda^{r}\left(V_{n-1}\right)+\frac{1}{2}\left\{V_{n-r+1}-V_{n-r-1}\right\} \Lambda^{r-1}\left(V_{n-1}\right) .
$$

The reason for the value of this form is provided by Lemma 2 above: it is elementary to write a computer program which shifts the subscripts of previous expansions the appropriate value up and down and then combines the results (allowing, of course, for the negative subscript cases changing to negative coefficients).

A different pair of recurrence relations is derivable from Lemmas 2 and 3 :

THEOREM 2. FOr $1<r \leq n-2, n \leq p$,

$$
\Lambda^{r}\left(v_{n}\right)=\left\{v_{r+1}-v_{r-1}\right\} \Lambda^{r}\left(v_{n-1}\right)-\Lambda^{r}\left(v_{n-2}\right)+\Lambda^{r-2}\left(v_{n-2}\right) .
$$

Proof. Expansion of the right hand side gives

$$
\begin{aligned}
& \frac{V_{n-2} \cdots V_{n-r+1}}{V_{r} \cdots V_{1}}\left\{V_{r} V_{r-1}-V_{n-r} V_{n-r-1}+V_{n-1} V_{n-r}\left[V_{r+1}-V_{r-1}\right]\right\} \\
& =\frac{V_{n-2} \cdots V_{n-r+1}}{V_{r} \cdots V_{1}}\left\{V_{n-1} V_{2 r-n}+V_{n-1}\left[V_{n}+V_{n-2 r}\right]\right\} \text { on applying Lemmas } 2 \text { and } 3 \\
& =\frac{V_{n-2} \cdots V_{n-r+1}}{V_{r} \cdots V_{1}} \cdot V_{n} \cdot V_{n-1} \\
& =\Lambda^{r}\left(V_{n}\right) .
\end{aligned}
$$

This recurrence relation is also in convenient form for computer programming.

The dual is not of great value, but is worth stating:

COROLLARY.

$$
\Lambda^{r}\left(v_{n}\right)=\left[V_{n-r+1}-V_{n-r-1}\right] \Lambda^{r-1}\left(V_{n-1}\right)-\Lambda^{r-2}\left(v_{n-2}\right)+\Lambda^{r}\left(V_{n-2}\right)
$$




\section{References}

[1] Gert Almkvist and Robert Fossum, "Decomposition of exterior and symmetric powers of indecomposable $\mathbb{Z} / p \mathbb{Z}$ modules in characteristic $p$ and relations to invariants", Séminaire d'Algèbre Paul Dubreil, 1-11l (Proceedings, Paris 1976-1977. Lecture Notes in Mathematics, 641. Springer-Verlag, Berlin, Heidelberg, New York, 1978).

[2] J.-C. Renaud, "The decomposition of products in the modular representation ring of a cyclic group of prime power order", J. Algebra 58 (1979), 1-11.

Department of Mathematics,

University of Papua New Guinea,

PO Box 320,

University,

Port Moresby,

Papua New Guinea. 